\section{Agreement and clinical comparison between a new swept-source optical coherence tomography-based optical biometer and an optical low-coherence reflectometry biometer}

\begin{abstract}
Purpose To compare measurements taken using a swept-source optical coherence tomography-based optical biometer (IOLmaster 700) and an optical low-coherence reflectometry biometer (Lenstar 900), and to determine the clinical impacts of differences in their measurements on intraocular lens (IOL) power predictions.

Methods Eighty eyes of 80 patients scheduled to undergo cataract surgery were examined with both biometers. The measurements made using each device were axial length (AL), central corneal thickness (CCT), aqueous depth (AQD), lens thickness (LT), mean keratometry (MK), white-to-white distance (WTW), and pupil diameter (PD).

Holladay 2 and SRK/T formulas were used to calculate IOL power. Differences in measurement between the two biometers were determined using the paired $t$-test. Agreement was assessed through intraclass correlation coefficients (ICC) and BlandAltman plots.

Results Mean patient age was $76.3 \pm 6.8$ years (range 59-89). Using the Lenstar, AL and PD could not be measured in $\mathbf{1 2 . 5}$ and $5.25 \%$ of eyes, respectively, while IOLMaster 700 took all measurements in all eyes. The variables CCT, AQD, LT, and MK varied significantly between the two biometers. According to ICCs, correlation between measurements made with both devices was excellent except for WTW and PD. Using the SRK/T formula, IOL power prediction based on the data from the two devices were
\end{abstract}

P Arriola-Villalobos ${ }^{1,2}$, J Almendral-Gómez', N Garzón ${ }^{3}$, J Ruiz-Medrano ${ }^{1}$, C Fernández-Pérez ${ }^{4}$, JM Martínez-de-la-Casa ${ }^{2,5}$ and D Díaz-Valle 2,5

statistically different, but differences were not clinically significant.

Conclusions No clinically relevant differences were detected between the biometers in terms of their measurements and IOL power predictions. Using the IOLMaster 700 , it was easier to obtain biometric measurements in eyes with less transparent ocular media or longer AL. Eye (2017) 31, 437-442; doi:10.1038/eye.2016.241; published online 11 November 2016

\section{Introduction}

Accurate calculation of intraocular lens (IOL) power is crucial for the success of cataract surgery. ${ }^{1}$ For this purpose, modern biometry methods such as ultrasound and optical biometry are used. The main optical biometry devices currently available are the IOLMaster 500 (Carl Zeiss Meditec AG, Jena, Germany) based on partial coherence interferometry, the Lenstar 900 (Haag-Streit AG, Koeniz, Switzerland), which uses optical low-coherence reflectometry (OLCR) and is powered by a superluminescent diode (SLD), and the recently developed IOLMaster 700 (Carl Zeiss Meditec AG, Jena, Germany) based on swept-source optical coherence tomography (SS-OCT) technology. Following its introduction over $>15$ years ago, the IOLMaster 500 has become the benchmark for optical biometry instruments, offering high precision and good resolution for axial length (AL), anterior chamber depth (ACD), and corneal keratometry measurements. ${ }^{2}$

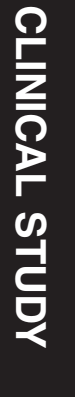

${ }^{1}$ Ophthalmology, Hospital Clínico San Carlos, Instituto de Investigación Sanitaria del Hospital Clínico San Carlos (IdISSC), Madrid, Spain

2Patología ocular del envejecimiento, calidad visual y calidad de vida, Instituto de Salud Carlos III, Madrid, Spain

Instituto de Oftalmología Avanzada, Madrid, Spain

${ }^{4}$ Investigative Support Unit, Hospital Clínico San Carlos, Instituto de Investigación Sanitaria del Hospital Clínico San Carlos (IdISSC), Madrid, Spain

${ }^{5}$ Ophthalmology, Hospital Clínico San Carlos, Departamento de Oftalmología y ORL, Facultad de Medicina, Universidad Complutense de Madrid, Instituto de Investigación Sanitaria del Hospital Clínico San Carlos (IdISSC), Madrid, Spain

Correspondence:

P Arriola-Villalobos, Ophthalmology, Hospital ClÃnico San Carlos, C/ Pez Volador $32,9^{\circ} \mathrm{D}, \mathrm{C} /$ Prof Martin Lagos s/n, Ciudad Universitaria, Madrid 28007, Spain

Tel: +0034913303132;

Fax: +0034913303975.

E-mail: pedro_arriola@

hotmail.com

Received: 11 April 2016 Accepted in revised form:

7 September 2016

Published online:

11 November 2016 
The Lenstar 900, which also measures lens thickness (LT) and central corneal thickness (CCT), has shown good reproducibility ${ }^{3}$ and good correlation with the IOLMaster $500 .{ }^{4}$ However, both devices have returned considerable $\mathrm{AL}$ measurement failure rates in eyes with mature or posterior subcapsular cataracts. ${ }^{5}$ The new IOLMaster 700, the first SS-OCT-based biometer, features excellent repeatability and reproducibility, and also high agreement with IOLMaster $500 .^{6}$

In two recent studies assessing agreement between Lenstar 900 and IOLMaster 700 measurements, 7,8 good agreement between both the devices was reported. However, to date, no study has compared IOL power predictions made using these two biometers.

This study sought to compare IOLMaster 700 and Lenstar 900 measurements, and to assess the possible clinical impacts of differences in these measurements on IOL power calculations.

\section{Patients and methods}

This was a randomized diagnostic technology assessment. Participants were consecutively recruited among patients evaluated for possible cataract surgery at our centre

Table 1 IOLMaster 700 and Lenstar LS 900 measurements (means $\pm \mathrm{SD}$ )

\begin{tabular}{lcc}
\hline Variable & IOLMaster 700 & Lenstar LS 900 \\
\hline $\mathrm{AL} / \mathrm{mm}$ & $23.536 \pm 1.54$ & $23.531 \pm 1.54$ \\
$\mathrm{CCT} / \mu \mathrm{m}$ & $544.74 \pm 34.45$ & $542.23 \pm 33.32$ \\
$\mathrm{AQD} / \mathrm{mm}$ & $2.54 \pm 0.34$ & $2.56 \pm 0.34$ \\
$\mathrm{LT} / \mathrm{mm}$ & $4.71 \pm 0.4$ & $4.67 \pm 0.38$ \\
$\mathrm{MK} / \mathrm{D}$ & $44.56 \pm 1.62$ & $44.62 \pm 1.63$ \\
$\mathrm{WTW} / \mathrm{mm}$ & $11.81 \pm 0.44$ & $11.82 \pm 0.59$ \\
$\mathrm{PD} / \mathrm{mm}$ & $3.87 \pm 0.81$ & $3.79 \pm 0.66$ \\
\hline
\end{tabular}

Abbreviations: $\mathrm{AL}$, axial length; $\mathrm{AQD}$, endothelium to lens distance; $\mathrm{CCT}$, central corneal thickness; D, diopters; LT, lens thickness; MK, mean keratometry; $\mathrm{PD}$, pupil diameter; WTW, white-to-white distance.
(Hospital Clinico San Carlos, Madrid, Spain). The inclusion criterion was age-related cataract (including mild cataract). Exclusion criteria were prior eye surgery, eyes with active disease, such as uveitis or retinal degeneration, and prior contact lens wear. If both eyes met the inclusion and exclusion criteria, the right eye was selectd for inclusion in the study. Only one eye of each subject was included since ocular measurements are more similar between fellow eyes than between eyes of different individuals. ${ }^{9}$ The study protocol adhered to the tenets of the Declaration of Helsinki and Spanish legislation, and was approved by our Institutional Clinical Research Ethics Committee. Before recruitment, written legally binding informed consent was obtained from each patient.

After a brief biomicroscopy examination without instillation of drops to confirm the diagnosis of cataract, patients were consecutively examined using the two optical biometers, IOLMaster 700 and Lenstar LS 900 (Eye Suite i4000 software) in random order. Subjects were examined between 8 am and 3 pm in a small office, centrally heated to a temperature of 21 to $25^{\circ} \mathrm{C}$, with diffuse lighting. There were no ventilation ducts over the equipment. Both instruments were placed side-by-side in the same room to reduce examination time. Both examinations were conducted by the same examiner (PAV), a consultant surgeon with more than ten years of experience in biometry. Subjects were asked to blink inmediately before measurements to ensure an optically smooth tear film over the cornea. IOLMaster 700 measurements were only accepted when validated by the device's inbuilt quality test. Lenstar 900 measurements were made according to the manufacturer's instructions, obtaining a minimum of three quality images. The data recorded were AL, CCT, aqueous depth (AQD) (endothelium to lens distance), LT, mean keratometry (MK), white-to-white distance (WTW), and pupil diameter (PD). The IOL power required for emmetropia using an A-constant of 118.0 with the Holladay 2 and

Table 2 Agreement of parameters measurements between the IOLMaster 700 and the Lenstar LS 900

\begin{tabular}{|c|c|c|c|c|c|c|}
\hline \multirow{2}{*}{ Variable } & \multirow{2}{*}{ Mean difference } & \multirow{2}{*}{ P-value } & \multirow{2}{*}{ ICC } & \multirow{2}{*}{$C I(95 \%)$} & \multicolumn{2}{|c|}{$95 \%$ LoA } \\
\hline & & & & & Lower & Upper \\
\hline $\mathrm{AL} / \mathrm{mm}$ & $0.0046 \pm 0.022$ & 0.086 & 1 & 1 to 1 & -0.04 & 0.05 \\
\hline $\mathrm{CCT} / \mu \mathrm{m}$ & $2.513 \pm 5.77$ & $<0.001$ & 0.983 & 0.974 to 0.989 & -9.03 & 14.05 \\
\hline $\mathrm{AQD} / \mathrm{mm}$ & $-0.015 \pm 0.038$ & 0.001 & 0.993 & 0.989 to 0.995 & -0.09 & 0.06 \\
\hline $\mathrm{LT} / \mathrm{mm}$ & $0.0353 \pm 0.099$ & 0.002 & 0.964 & 0.944 to 0.977 & -0.16 & 0.23 \\
\hline $\mathrm{MK} / \mathrm{D}$ & $-0.0546 \pm 0.17$ & 0.006 & 0.994 & 0.99 to 0.996 & -0.39 & 0.28 \\
\hline $\mathrm{WTW} / \mathrm{mm}$ & $-0.01 \pm 0.417$ & 0.831 & 0.682 & 0.545 to 0.783 & -0.84 & 0.82 \\
\hline $\mathrm{PD} / \mathrm{mm}$ & $0.085 \pm 0.538$ & 0.175 & 0.733 & 0.608 to 0.822 & -0.99 & 1.16 \\
\hline
\end{tabular}

Abbreviations: ACD, endothelium to lens distance; AL, axial length; CCT, central corneal thickness; CI, confidence interval; ICC, intraclass correlation coefficient; LoA, limits of agreement; LT, lens thickness; MK, mean keratometry; PD, pupil diameter; WTW, white-to-white distance. 
SRK/T IOL formulas was calculated after the examination for each eye using the two sets of measurements.

For a descriptive statistical analysis, we used Excel 2011 (Microsoft Corp. Redmond, WA, USA) with SPSS software (version 18.0, SPSS Inc.). Results are reported as mean \pm SD. Significance was set at $P \leq 0.05$. The data were compared using a paired-sample $t$-test. Consistency between the biometers was assessed by determining intraclass correlation coefficients (ICC) at the $95 \%$ confidence level. Bland-Altman plots were also constructed to compare the devices. ${ }^{10}$ Proportion of differences falling within the clinically acceptable ranges defined by Jasvinder et al ${ }^{11}$ were also estimated. These ranges are IOL power within 1 diopter (D), $1-2 \mathrm{D}$ or > 2D; AL within $0.33 \mathrm{~mm}$ or within $0.10 \mathrm{~mm}$; and MK within $1 \mathrm{D}$ or within $0.5 \mathrm{D}$. In addition, $95 \%$ limits of agreement (LoA) were calculated for each measurement, computed as the mean $\pm 2 \mathrm{SD}$ of the difference between both devices.

\section{Results}

The study sample consisted of 80 eyes (71 right eyes) of 80 patients ( 34 men, 46 women) of mean age $76.3 \pm 6.8$ years (range: 59-89). With the Lenstar 900, AL could not be measured in 10 of the eyes $(12.5 \%)$ because of dense cataract (8 eyes) or an AL longer than $30 \mathrm{~mm}$ (2 eyes, $20 \%)$, and PD could not be measured in 5 eyes (6.25\%). Good quality check images were nevertheless saved. With the IOLMaster 700, a complete set of measurements were obtained in each eye.

Of the variables determined with both biometers (Table 1), significant differences were recorded in CCT, AQD, LT, and MK. Differences in the AL data approached significance.

Table 3 IOL power (in diopters) predictions in IOLMaster 700 and Lenstar LS 900

\begin{tabular}{lcl}
\hline Formula & IOLMaster 700 & Lenstar 900 \\
\hline Holladay 2 & $18.77 \pm 3.92$ & $18.74 \pm 3.9$ \\
SRK/T & $19.12 \pm 3.77$ & $19.07 \pm 3.75$ \\
\hline
\end{tabular}

Good correlation (ICCs of 0.96-1.00) was observed for all variables determined with both devices except WTW and PD (Table 2).

Calculated IOL powers (Table 3) differed significantly between the biometers when the SRK/T formula was used, but were similar using the Holladay 2 formula. Agreement in IOL power predictions based on the measurements obtained using both biometers was excellent (Table 4).

Our Bland-Altman plots indicate measurement differences between biometers and differences in IOL power calculations (Figure 1), along with their 95\% LoA.

When clinically acceptable ranges were examined, all AL measurements were within $0.10 \mathrm{~mm}$; in 79 eyes the MK data were within $0.5 \mathrm{D}$ (all within 1D); in all 80 eyes, IOL powers predicted with the SRK/T formula were within $1 \mathrm{D}$, while only one eye showed a predicted IOL power using the Holladay 2 formular, varying only by 1-2 D (1.1 D).

\section{Discussion}

The findings of our study indicate significant differences in several variables measured using the IOLMaster 700 and Lenstar 900, and that these differences led to different IOL power predictions according to the SRK/T formula. The latter differences, nevertheless not clinically significant, and in only one eye did the power predicted with the Holladay 2 formula show a difference $>1 \mathrm{D}$. The differences detected are likely the result of the devices' different technologies: the Lenstar 900 is based on OLCR powered by a SLD and the IOLMaster 700 uses SS-OCT technology. However, their agreement between most measurements was excellent, with the exception of WTW and PD. The IOLMaster 700 was more successful at obtaining AL measurements in patients with dense cataracts or long eyes (longer than $30 \mathrm{~mm}$ ).

There was full correlation in AL measurements between the devices (ICC $=1$ ); the mean difference was scarcely $0.0046 \mathrm{~mm}$ and the agreement was excellent $(95 \%$ LoA). In other studies in which agreement between Lenstar 900 and IOLMaster 500 was assessed, 3,4,12,13 AL was always higher with the Lenstar, whereas in our study

Table 4 Agreement of IOL power (in diopters) predictions between the IOLMaster 700 and the Lenstar LS 900

\begin{tabular}{lcccccc}
\hline Formula & Mean difference & P-value & ICC & CI (95\%) & 95\% LoA \\
\cline { 3 - 6 } & & & & & Lower \\
\hline Holladay 2 & $0.034 \pm 0.257$ & 0.272 & 0.998 & 0.997 to 0.999 & -0.51 \\
SRK/T & $0.0517 \pm 0.186$ & 0.023 & 0.999 & 0.998 to 0.999 & -0.32 & 0.58 \\
\hline
\end{tabular}

Abbreviations: CI, confidence interval; ICC, intraclass correlation coefficient; LoA, limits of agreement. 
a

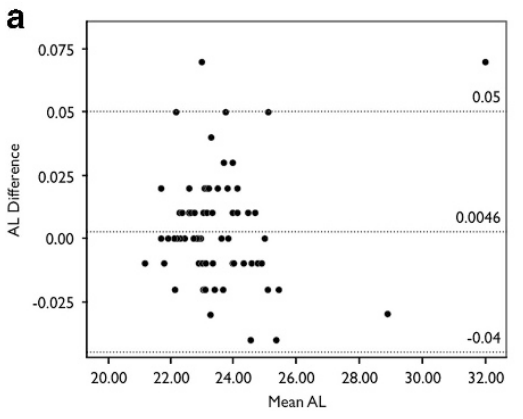

d

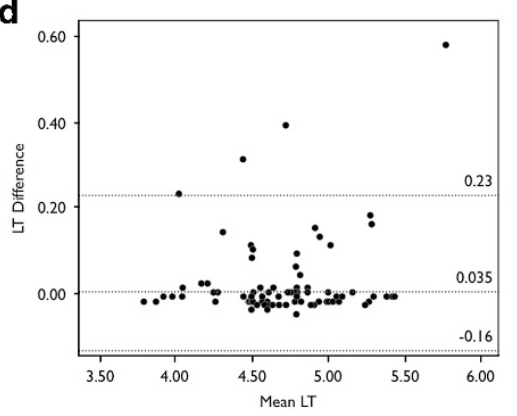

g

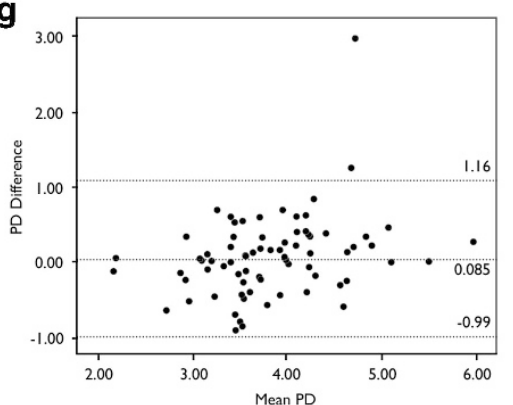

b

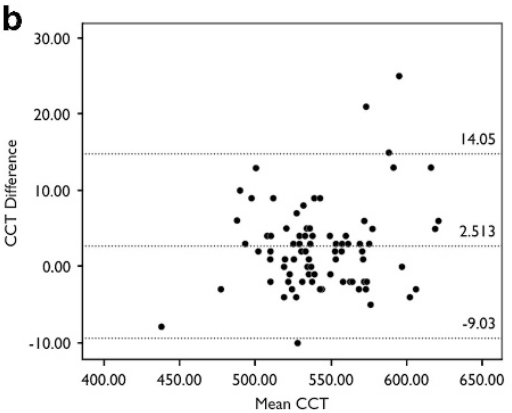

e

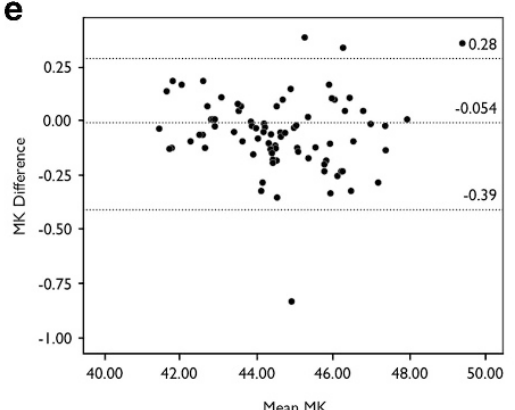

h

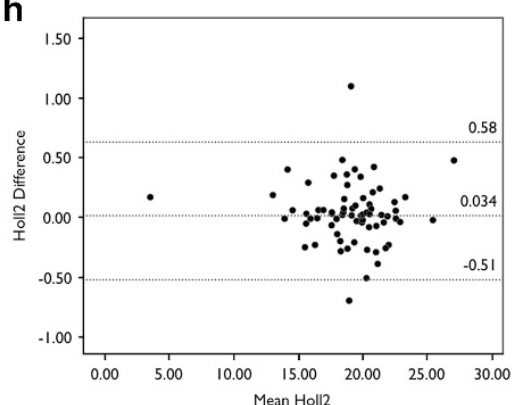

C

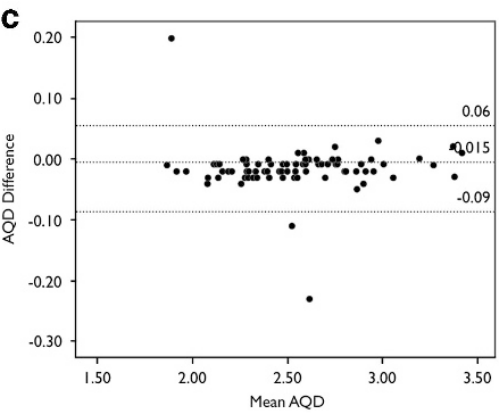

f

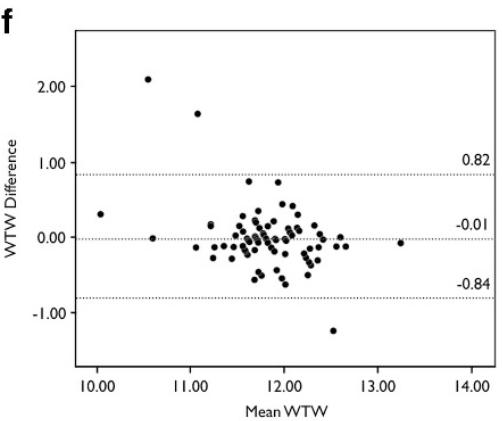

i

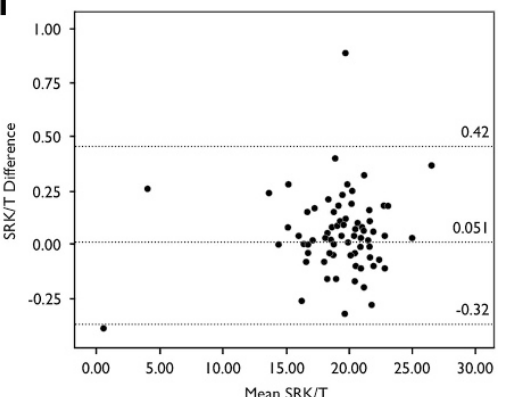

Figure 1 Bland-Altman plots showing agreement between measurements taken with IOLmaster 700 and Lenstar 900 . The middle line shows the mean difference, and the bottom and top lines show the lower and upper $95 \%$ limits of agreement, respectively. (a) AL measurements; (b) CCT measurements; (c) AQD measurements; (d) LT measurements; (e) MK measurements; (f) WTW measurements; (g) PD measurements; (h) IOL power calculated with the Holladay 2 formula, and (i) IOL power calculated with the SRK/T formula.

AL was slightly longer when measured with IOLMaster 700 .

Aside from AL error, keratometry reading error is one of the most common causes of preoperative IOL power error resulting in the need for postoperative lens exchange. ${ }^{14}$ Again, there was excellent correlation in MK measurements between the devices examined here (ICC $=0.994)$, although there was a significant difference of barely $0.05 \mathrm{D}$. Good correlation between these variables was also reported in the studies mentioned. $3,4,12,13$

We also observed good correlation (according to ICCs and LoA) in CCT, AQD, and LT measurements. As for the MK data, these minor, yet significant, differences seem have little consequence on IOL calculations. The SRK/T formula bases its calculations on AL and MK measurements. In our study, the significant difference between the two devices for SRK/T formula IOL power calculation was only $0.05 \mathrm{D}$, while the $95 \%$ LoA confirmed the high level of agreement. In effect, all eyes showed a predicted IOL power within $1 \mathrm{D}$ using the SRK/T formula. The Holladay 2 is a fourth-generation formula based on variables such as LT, AQD, and WTW added to AL and MK for IOL power calculation. No significant differences emerged between both biometers when IOL powers were calculated with the Holladay 2 formula. However, one eye showed an IOL power prediction difference of $1.1 \mathrm{D}$ depending on the device 
used due mainly to the differences in MK and WTW measurements.

It was not possible to obtain $\mathrm{AL}$ measurement in 10 $(12.5 \%)$ of the eyes using Lenstar 900: in 2 eyes $(2.5 \%)$ because they were longer than $30 \mathrm{~mm}$, and in $8(10 \%)$ because they had dense cataracts. This percentage of $12.5 \%$ is similar to the proportions of unsuccessful scans due to opaque ocular media reported for the Lenstar 900 in other studies (Buckhurst et $a l^{3} 10 \%$, and Roher et al $11.1 \%$ ), yet higher than the rate provided by others (Chen et $a^{15} 6 \%$ ). In contrast, the IOLMaster 700 provided reliable measurements in all eyes, even in the two eyes longer than $30 \mathrm{~mm}$. This indicates that that the IOLMaster 700 is more effective than the Lenstar in obtaining biometric measurements not just in eyes with less transparent ocular media, but also in eyes with longer AL. This last issue, which has not been reported elsewhere, may be explained by the SS-OCT technology and is relevant in the clinical practice. Furthermore, unusual eye geometries, such as a tilt or decentration of the crystalline lens, can be detected. The fovea imaging offered by this instrument also serves to detect poor fixation and possible retinal abnormalities such that patients may be then referred to a retina specialist. ${ }^{6}$

As limitations of our study, we should first mention that our study sample did not include short eyes $(<21.0 \mathrm{~mm})$, for which different results might be observed. Secondly, both sets of measurements were made by the same examiner, who was therefore not blind to the other device's measurement. Finally, we did not conduct repeatability tests, though the repeatability of both Lenstar 900(ref. 3) and the IOLMaster 700 (refs 6-8) measurements has proved excellent.

The patients for our study were recruited from a continuous cohort. As inclusion criteria were not strict, our study population as far as possible represents patients examined in routine clinical practice. Our aim was to not limit comparisons by including only patients with similar cataract severities. Neither did we adjust for potential effects of age nor for gender.

To the best of our knowledge, this is the first study to compare the IOL power predictions based on the measurement provided by Lenstar 900 vs the new IOLMaster 700.

In conclusion, good agreement was observed for most ocular biometry measurements, and IOL predictions made using IOLMaster 700 and Lenstar 900. Despite several significant differences in measurements between the two instruments, none of these were considered clinically relevant. The IOLMaster 700 was more effective at obtaining biometric measurements in longer eyes and eyes with less transparent ocular media.

\section{Summary}

\section{What was known before}

- The Lenstar 900 biometer has shown good reproducibility but has a significant measurement failure rate in mature or posterior subcapsular cataracts.

- The new IOLMaster 700 has shown excellent repeatability and reproducibility.

What this study adds

- The new IOLMaster 700 showed good agreement with the Lenstar in most of the ocular biometry measurements. The calculated IOL powers from the two biometers were similar.

- The new IOLMaster 700, due to the SS-OCT technology, was more effective in obtaining AL measurements in cases of dense cataract or long AL.

\section{Conflict of interest}

The authors declare no conflict of interest.

\section{References}

1 Olsen T. Calculation of intraocular lens power: a review. Acta Ophthalmol Scand 2003; 85: 472-485.

2 Drexler W, Findl O, Menapace R, Rainer G, Vass C, Hitzenberger CK et al. Partial coherence interferometry: a novel approach to biometry in cataract surgery. $A m \mathrm{~J}$ Ophthalmol 1998; 126: 524-534.

3 Buckhurst PJ, Wolffsohn JS, Shah S, Naroo SA, Davies LN, Berrow EJ. A new optical low coherence reflectometry device for ocular biometry in cataract patients. Br J Ophthalmol 2009; 93: 949-953.

4 Roher K, Frueh BE, Wälti R, Clemetson IA, Tappeiner C, Goldblum D. Comparison and evaluation of ocular biometry using a new noncontact optical low-coherence reflectometer. Ophthalmology 2009; 116: 2087-2092.

5 McAlinden C, Wang Q, Pesudovs K, Yang X, Bao F, Yu A et al. Axial length measurement failure rates with the IOLMaster and Lenstar LS 900 in eyes with cataract. PLoS One 2015; 10: e0128929.

6 Srivannaboon S, Chirapapaisan C, Chonpimai P, Loket S. Clinical comparison of a new swept-source optical coherence tomography-based optical biometer and a time-domain optical coherence tomography-based optical biometer. J Cataract Refract Surg 2015; 41: 2224-2232.

7 Kunert KS, Peter M, Blum M, Haigis W, Sekundo W, Schütze J et al. Repeatability and agreement in optical biometry of a new swept-source optical coherence tomography-based biometer versus partial coherence interferometry and optical low-coherence reflectometry. J Cataract Refract Surg 2016; 42: 76-83.

8 Kurian M, Negalur N, Das S, Puttaiah NK, Haria D, J TS et al. Biometry with a new swept-source optical coherence tomography biometer: Repeatability and agreement with an optical low-coherence reflectometry device. J Cataract Refract Surg 2016; 42: 577-581.

9 Katz J, Zeger S, Liang K-Y. Appropriate statistical methods to account for similarities in binary outcomes between fellow eyes. Invest Ophthalmol Vis Sci 1994; 35: 2461-2465. 
10 Bland JM, Altman DG. Statistical methods for assessing agreement between two methods of clinical measurement. Lancet 1986; 1: 307-310.

11 Jasvinder S, Khang TF, Sarinder KK, Loo VP, Subrayan V. Agreement analysis of LENSTAR with other techniques of biometry. Eye (Lond) 2011; 25: 717-724.

12 Holzer MP, Mamusa M, Auffarth GU. Accuracy of a new partial coherence interferometry analyser for biometric measurements. Br J Ophthalmol 2009; 93: 807-810.
13 Hoffer KJ, Shammas HJ, Savini G. Comparison of 2 laser instruments for measuring axial length. J Cataract Refract Surg 2010; 36: 644-648.

14 Jin GJC, Crandall AS, Jones JJ. Intraocular lens exchange due to incorrect lens power. Ophthalmology 2007; 114: 417-424.

15 Chen YA, Hirnschall N, Findl O. Evaluation of 2 new optical biometry devices and comparison with the current gold standard biometer. J Cataract Refract Surg 2011; 37: 513-517. 